•综述・

\title{
动物移动网络研究对景观生态学的贡献
}

\author{
李月辉๑, 胡远满๑*
}

中国科学院沈阳应用生态研究所中国科学院森林生态与管理重点实验室, 沈阳 110016

摘要: 景观生态学在其发展之初的 20 世纪 80 年代, 提出了关于景观网络研究(包括景观网络概念、网络结构指数和景观连接 度)的基本构想, 这些构想需要在景观过程的研究中逐渐被落实和发展。动物移动过程因动物在斑块或廊道上有着独特丰富 的属性特征、与周围资源环境之间存在复杂反馈而区别于无机物运移的景观过程, 则动物移动网络研究在实现关于景观网络 研究的基本构想、推动景观生态学发展中贡献独特。因此, 总结动物移动网络研究的来源脉络及其对景观生态学的理论贡献 对于景观网络领域和景观生态学学科的发展都具有重要意义。本文抓住景观生态学发展之初提出的关于景观网络研究的基本 构想, 寻找和剖析其中所蕴含的景观生态学思想, 追踪这些思想如何被落实、发展、并形成目前的三个热点方向: 动物移动 网络模拟、重要值评价和景观连接度分析; 总结这三个方向的研究进展, 指出整合动物的空间行为特征是必然发展趋势; 揭 示出动物移动网络研究始终都以发掘斑块或廊道的动物有机体的属性特征(如种群数量)、以及描述这种属性在不同斑块或廊 道之间的差异和联系为方向, 正是这种属性的发掘有效地落实、发展和丰富了关于景观网络研究的最初构想, 对景观生态学 的贡献比其他过程更为独特。文章还总结了我国动物移动网络研究与国际研究相比较为滞后的现状, 指出其暂时尚未显示出 对我国景观生态学的独特贡献; 强调发展源于跟踪定位数据的动物空间行为生态学研究是减小差距的重要、必要前提。期望 本文能引发关于景观网络乃至景观生态学理论发展的方向性思考, 为研究者提供参考。

关键词: 动物移动网络; 景观生态学; 景观格局; 景观过程; 景观功能; 迁徙网络模拟; 网络要素重要值; 景观连接度; 空间 移动行为

李月辉, 胡远满 (2021) 动物移动网络研究对景观生态学的贡献. 生物多样性, 29, 98-108. doi: 10.17520/biods.2020219.

$\mathrm{Li} \mathrm{YH,} \mathrm{Hu} \mathrm{YM} \mathrm{(2021)} \mathrm{The} \mathrm{contributions} \mathrm{of} \mathrm{animal} \mathrm{movement} \mathrm{network} \mathrm{researches} \mathrm{to} \mathrm{landscape} \mathrm{ecology.} \mathrm{Biodiversity} \mathrm{Science,} \mathrm{29,} \mathrm{98-108.} \mathrm{doi:}$ 10.17520/biods.2020219.

\section{The contributions of animal movement network researches to landscape ecology}

Yuehui $\mathrm{Li}^{(\mathbb{D})}$, Yuanman $\mathrm{Hu}^{(\mathbb{D} *}$

CAS Key Laboratory of Forest Ecology and Management, Institute of Applied Ecology, Chinese Academy of Sciences, Shenyang 110016

\begin{abstract}
Context: As early as the emergence of landscape ecology in the 1980s, scientists began to conceive ideas about landscape networks including landscape networks concepts, network metrics, and landscape connectivity. Those early ideas needed to be gradually realized through the exploration of concrete landscape processes. Animal movement processes are substantially different from inorganic processes due to the fact that animals provide a variety of biological attributes in each corridor or patch and feed back into their habitat in a highly complex way. Therefore, the animal movement network research especially contribute to realizing and developing those early ideas to build up landscape network research.

Objectives: We present a review to summarize the development of research focusing on animal movement networks and how it has contributed to landscape ecology. We also provide an overview of animal movement network research progress in China and recognize the gap between China and developed countries.
\end{abstract}

收稿日期: 2020-05-27; 接受日期: 2020-08-17

基金项目: 国家自然科学基金(41871197; 41271201)和国家重点研发计划(2016YFC0500401)

* 通讯作者 Author for correspondence. E-mail: huym@iae.ac.cn 
Results and conclusions: We summarize the early ideas of landscape networks including landscape networks concepts, network metrics, and landscape connectivity which have shaped three current focuses in animal network research: modelling movement networks, evaluating importance of network elements, and examining functional landscape connectivity. It is considered as the trend of incorporating animal movement to movement network research. We illustrate that the research on movement networks has aimed at quantifying biological attributes in each corridor/patch as well as the connections or differences among corridors/patches in those attributes. This has made a special contribution to realizing those early ideas and developing landscape ecology from the era of focusing on only inorganic processes. Also, we highlight that there is a gap in animal movement networks research between China and developed countries, which hinders the special contributions to landscape ecology study in China. Additionally, we emphasize the importance and necessity of movement ecology research employing GPS tracking data to improve knowledge of animal movement networks and narrow the research gap.

Key words: animal movement network; landscape ecology; landscape patterns; landscape processes; landscape function; simulation of migratory network; importance of network elements; landscape connectivity; movement behavior

景观生态学作为一门有理论和实践的学科, 在 20 世纪80年代开始出现和发展(Forman, 1995), 认 为景观由“廊道-斑块-基质”构成, 纵横交错的廊道 及廊道所连接的斑块构成景观网络, 提出了关于景 观网络概念、网络结构指数及景观连接度的基本构 想(Forman \& Godron, 1986)。这些构想体现出当时 对景观网络的认识限于几何属性, 对功能属性的认 识尚在萌芽; 同时, 也蕴含了落实和发展这些构想 的研究方向, 即描述景观网络的功能属性及其差异 和联系。景观网络的功能属性要在具体的景观过程 中体现。目前所研究的景观过程可以归纳为四类: 水过程(水及养分运移等)、土壤过程(土壤养分及污 染物运移等)、大气过程(大气污染物运移等)和生物 过程(动物的移动、种子和病毒的传播等)。前三类 过程形成的网络其功能属性是无机物的特征, 例如 土壤养分的含量和组成; 在第四类过程中, 动物移 动形成的网络其功能属性是动物有机体的多水平 特征, 例如种群的数量、出生率、死亡率和性别组 成等, 是独特、丰富和变化的。这些独特丰富的功 能属性使得动物移动网络的研究空间更大, 例如斑 块上的鸟类种群数量动态可以用来评价该斑块在 网络中的重要值(Runge et al, 2006)。所以, 相比其 他过程形成的网络, 动物移动网络的研究在实现和 发展景观网络最初构想的过程中作用更大。

动物移动网络研究开始于1990年前后(Turner, 1989; Taylor et al, 1993), 至2000年后, GPS跟踪技 术快速发展起来, 能够揭示陆生动物及鸟类的迁徙 网络(Panzacchi et al, 2016; Van Toor et al, 2018; Cooper et al, 2019); 图论(Graph theory)的应用更深
入, 数据处理能力更强大, 能够评价网络中各要素 的重要值(Erickson et al, 2017; Wiederholt et al, 2018; Sample et al, 2019)、度量景观功能连接度(Finch et al, 2017; Cohen et al, 2018; Sarà et al, 2019)。这些研究 不仅落实了关于景观网络概念、网络结构指数和景 观连接度的最初构想, 而且不断创新, 推动了景观 网络理论的进步, 为“格局-过程”关系这一核心理 论的发展做出了独特贡献。

因此, 总结动物移动网络研究的发展脉络及其 对景观生态学的理论贡献对于景观网络研究领域 及景观生态学学科的发展都具有重要意义。本文阐 述关于景观网络的概念、网络结构指数和景观连接 度的基本构想及它们如何被落实、发展并形成目前 的三个热点方向; 总结这三个方向的研究进展, 凸 显未来发展趋势, 揭示动物移动网络研究对景观生 态学的理论贡献; 对比国际研究, 总结我国的研究 现状, 寻找与国际水平之间存在差距的原因。本文 试图引发关于景观网络乃至景观生态学理论发展 的方向性的思考, 为研究者提供参考。

\section{景观生态学对景观网络研究的最初构想}

\section{1 景观网络的概念}

在今天看来, 景观中的网络有 “结构网络” 和 “功能网络”之分, 前者是可见的“有形”的网络, 例 如道路、树篱和河流网络等, 后者是不可见的“无 形” 的网络(可以通过跟踪数据处理或者模型模拟在 图上展示出来), 是由各种景观过程形成的网络, 例 如污染物运移或动物运动形成的网络。“结构网络” 和“功能网络” 有时会部分甚至全部重合, 例如, 动 
物有时沿着道路移动, 道路结构网络和动物移动功 能网络部分重合; 车辆始终在道路上行驶, 道路结 构网络和车流功能网络完全重合。

景观生态学在其发展之初, 清楚地认识到了 “结构网络”, 明确提出了廊道和网络的概念: 廊道 是不同于两侧基质的线状或带状结构, 连通或者阻 碍物质和能量的流动; 廊道相互交叉形成网络, 更 错综复杂地与周围斑块或基质相互作用(Forman \& Godron, 1986; 邬建国, 2000)。同时, 也一定程度地 认识到了 “功能网络”, 因为同一时期形成了 “景观 格局和过程关系”这一核心理论, 识别和定义了景 观过程, 认为景观过程是物质、能量或信息的流动 (Risser et al, 1984; Forman \& Godron, 1986; Turner, 1989), 意味着必然涉及到由“流”而形成的廊道和网 络。只是, 该认识尚不清晰, 未明确提出相关概念。

景观网络概念的提出标志着景观网络理论将 成为景观生态学的重要组成部分, 对 “结构网络”的 清晰认识和对“功能网络”的萌芽认识都将在日后各 种景观过程的研究中被逐渐落实和深化, 成为景观 网络研究的重要基础内容, 即揭示和定量评价景观 过程形成的“功能网络”。

\section{2 景观网络结构指数}

景观生态学发展之初, 针对“可见”的结构性的 廊道和网络, 探索其结构和功能两方面特征: 一方 面，提出借用图论中的部分指数度量空间几何特征， 针对独立廊道, 有宽度、曲度和间断情况等指数; 针对网络, 有 $\gamma 、 \alpha$ 及网格大小等指数(Forman \& Godron, 1986)。这些指数都比较简单, 计算案例也 很少，等待在实践中被赋予更多的生态学属性和意 义。另一方面, 评价廊道或网络的功能属性的思想 开始萌芽, 例如树篦的物种组成和植被高度影响风 速(Forman \& Baudry, 1984)。这些评价思想需要在日 后各种景观过程的研究中赋予斑块或廊道相应的 属性之后来实现, 例如在污染物运移过程中斑块对 污染物的截留和清除功能(李秀珍等, 2001), 就不仅 可以依据斑块或廊道之间可见的空间关系, 还可以 依据相应的属性来度量和评价廊道和网络。

\section{3 景观连接度}

连接度的概念最初由Merriam引入景观生态学, 度量景观空间结构单元相互之间的连续性(Merriam, 1984; 张娜, 2014)。之后, 又被区分出结构连接度 (structural connectivity) 和功能连接度 (functional connectivity) (Baudry \& Merriam, 1988), 前者指景 观在空间上直接表现出的几何连续性，无关于任何 过程信息，通过空间计算即可获得; 后者指在结构 基础上以所研究的景观过程的特征尺度来确定的 景观连续性，例如种子传播的距离和动物移动的范 围等(Forman \& Godron, 1986; 邬建国, 2000)。

景观生态学在发展之初提出了景观功能连接 度的概念, 表明该学科强调网络节点或廊道具有功 能属性且功能属性在节点或廊道之间是有差别和 联系的。不同景观过程形成的节点或廊道有不同的 属性, 功能连接度研究的进步就体现在考虑了哪种 功能属性, 以及多大程度地考虑这种功能属性。但功 能属性如何表达，由于当时对景观过程的研究很浅 而尚不清楚, 后来随着过程研究的深入才逐渐明晰。

\section{2 动物移动网络研究的现状、趋势及对景 学发展的贡献}

上述关于景观网络的概念、网络结构指数和景 观连接度这三方面构想的落实方向都指向一个“焦 点”：网络中节点或廊道不仅具有几何属性，还具 有功能属性，描述和度量这种功能属性及其差异和 联系是景观网络研究的必然趋势。由于动物在节点 或廊道上有着独特、丰富和多变的多个水平的属性, 如种群的数量和性别组成等, 动物移动网络比土壤 养分流动等无机过程网络能够更充分地满足发展 “焦点”的需求, 为景观网络研究提供了巨大的空间。

景观网络的基本构想在动物移动网络研究中 落实和发展，并形成了目前的研究热点：网络的构 建和模拟、网络要素的重要性评价和景观连接度分 析，体现出发掘斑块或廊道的动物属性特征以及描 述该属性在斑块或廊道间的差异和联系始终是移 动网络研究的发展方向。正是对这种属性的发掘, 逐渐落实、发展和丰富了景观生态学建立之初的构 想, 对景观生态学的贡献比其他过程更为独特。

\section{1 动物移动网络的构建和模拟}

最初的“功能网络”概念在动物移动过程研究中 被逐渐发展和落实,目前主要体现在构建和模拟动 物移动网络这一内容中。学者们从研究陆生动物开 始, 利用景观特征来模拟它们移动的潜在廊道网 络。最常用的模型有最小费用路径模型(least-cost path models)和电路模型(current models), 前者通过 赋予阻力值计算阻力面, 构建出移动阻力最小的潜 
在廊道(LaRue \& Nielsen, 2008; Watts et al, 2010; Dutta et al, 2016), 后者将景观表面视为导体计算其 电阻的大小而构建潜在廊道(Adriaensen et al, 2003; Blazquez-Cabrera et al, 2019; Vanthomme et al, 2019)。在能够考虑目标种的实际空间行为特征之前, 此类模拟方法是揭示动物移动网络、为动物管理提 供依据的重要手段(Watts et al, 2010)。

但是, 仅依据景观特征模拟出的潜在廊道与动 物利用的实际廊道是有差别的。对于物理性质的无 机过程, 模拟出的流动廊道和实际流动廊道基本相 符, 例如水文模型根据栅格的高程、植被和土壤等 特征就能够判断出水流的实际方向; 而对于动物移 动过程, 潜在廊道是否被真正利用还决定于动物的 生理或心理状态(Lapoint et al, 2013; Keeley et al, 2017)。因为不了解该状态及该状态产生的结果, 即 动物的实际运动特征, 上述模型只能对此做出假设: 最小费用路径模型假设动物对景观具有整体的认 识, 这样动物会根据某些标准例如生境质量等辨识 出最优的路线, 并沿着该路线运动; 电路模型则假 设动物 “醉汉 (drunkards)”似地完全随机运动, 这是 不现实的两个极端的假设(Panzacchi et al, 2016)。解 决该问题的唯一途径是整合动物的运动特征。2000 年以后GPS跟踪技术发展起来, 极大地推进了动物 移动特征的研究, 由此, 动物移动网络模拟获得了 巨大的发展空间: 一方面, 能计算出动物运动的范 围、最大运动距离等实际运动特征, 完善最小费用 路径模拟结果, 例如确定廊道的宽度; 另一方面, 能向以运动轨迹为核心的路径模拟方向发展, 目前 在小尺度上已有案例, 例如移步选择方程模型(step selection function, SSF) (Panzacchi et al, 2016; Zeller et al, 2016; Martin et al, 2018)。可以说, 整合动物空 间行为学特征是陆生动物移动网络构建和模拟的 必然发展趋势 (Driezen et al, 2007; Pullinger \& Johnson, 2010; Zeller et al, 2014)。

2000年之后, 鸟类的迁徙网络构建和模拟案例 逐渐出现, 近几年成为研究热点。迁徙鸟类在越冬 地、停歇地和繁殖地之间长距离飞行, 构建它们的 迁徙网络时, 首先要分别识别出在越冬、中途停歇 和繁殖期间的栖息地斑块: 在繁殖期和越冬期, 可 直接利用核密度分析或者最小多边形法估算栖息 地; 在停歇期, 一般根据停留时间确定停歇地, 例 如 7 天、14 天等，目前主要利用社区发现法 (community detection) 或第一通过时间模型法 (first-passage time, FPT) (Buhnerkempe et al, 2016; Deng et al, 2019; Cooper et al, 2019)。接下来, 要构 建斑块之间的廊道: 不同于陆生动物，鸟类在栖息 地斑块之间的飞行被默认为直线, 以直线廊道连 接。在构建实际迁徙路线的基础上，一个重要的发 展趋势是模拟种群水平的迁徙网络, 可以通过贝叶 斯模型 (Bayesian model) 和布朗桥运动模型 (Brownian bridge movement models)等多种模拟方 法来实现(Palm et al, 2015; Guillemain et al, 2017), 并且要将栖息地斑块的景观特征和目标种类飞行 特征相互结合(Van Toor et al, 2018)。

至此, 可以看出, 动物移动网络的模拟实现了 当初“功能网络”概念的设想, 并且从单纯考虑地面 景观特征向兼顾动物空间行为学特征方向发展，使 得在实际景观中展示“功能网络”的研究内容更加丰 富, 比其他过程对景观生态学的贡献更为独特。

\section{2 动物移动网络的重要值评价}

自景观生态学发展之初提出借用图论中的指 数来度量景观网络之后, 这些指数逐渐被应用于多 个案例。2000年前后，图论应用更加深入，计算机 技术更加快速发展, 为动物移动网络特征评价带来 变革性的变化，即有能力将之前的多种指数综合起 来、构造并计算出更复杂的有生态学意义的空间关 系指数(Webster et al, 2002)。具体而言, 当节点和廊 道交互成网络，对整个网络进行评价时，之前针对 单独特征的指数就被综合成一个指标，即这些节点 或廊道在整个网络中的重要程度。例如, 原来针对 廊道的 $\beta$ 指数只表示与每个交点相连接的廊道的数 量, 此时就可以和其他指数相互结合在一起, 共同 决定网络节点的重要值。重要值着重表达各网络要 素在结构属性和功能属性上的差异, 动物在多个生 境中栖息、在多个生境之间移动，理解这些离散生 境的差异和相对重要性或者贡献率对生态学理论 发展(Kneitel \& Miller, 2003; Kerr et al, 2006)和物种 保护管理都意义重大(Thogmartin, 2010; Sheehy et al, 2011; Wiederholt et al, 2018)。

重要性的评价包括结构和功能两方面, 结构要 素的重要性针对动物移动网络的几何特征, 落实了 当初网络指数评价的构想。目前多用节点中心度 (centrality)来确定节点在保持网络连接性中的重要 性(Estrada \& Bodin, 2008; Iwamura et al, 2014), 常 
用的具体指标有 3 个: 节点特征向量中心度 (eigenvector centrality); 节点中介中心性 (betweenness), 表示通过目标点的最短路径的数量; 节点连接中心度(node degree), 表示目标节点与其 他节点的连接强度(Nicol et al, 2016; Buhnerkempe et al, 2016; Knight et al, 2018)。

评价动物移动网络要素的功能重要性, 目前的 研究热点是利用种群动态这一功能属性, 也就是动 物在不同季节所利用的斑块在整个迁徙种群动态 中的相对重要性(Pulliam, 1988; Erickson et al, 2017), 是当初景观网络功能属性评价的萌芽思想的发展。 与迁徙的陆生哺乳动物相比, 鸟类迁徙网络的重要 值研究更多, 成为热点中的热点, 原因是鸟类的环 志数据或跟踪数据较多, 更有条件估算节点的种群 特征(Taylor \& Norris, 2010)。

事实上, 不同斑块上局部种群的重要性是复合 种群研究始终关注的重要内容(Hanski, 1998; Taylor \& Hall, 2012), 该内容和景观生态学思想相互融合 之后, 学者们开始在更大的空间尺度上评估这种重 要性, 并发展出多种评价指数。Runge等(2006)针对 非迁徙但是具有空间结构异质性的种群提出了 $C^{\mathrm{r}}$ 指 数, 即利用 $r$ 生境斑块中动物种群个体对产生下一 代的贡献率来表达该生境斑块的相对重要值。 Wiederholt等(2018)又将 $C^{r}$ 指数应用于迁徙鸟类, 评 估两个迁徙季(如越冬期和繁殖期)斑块的重要性。 后来又发展出了基于迁徙路径的指数 (pathway-based metric), 可以评估多个迁徙季斑块 的重要性(Erickson et al, 2017; Sample et al, 2019)。 但此类指数对数据要求高, 需要每一个节点上的种 群数量动态特征, 并非所有节点都满足需要, 有时 不得不利用模型来模拟这些特征(Bieri et al, 2018), 因此, 此类应用案例在近几年才开始出现, 有时还 不得不使用模拟数据(Wiederholt et al, 2018)。尽管 如此, 以鸟类迁徙轨迹及鸟类种群在节点上的属性 为核心的研究是必然发展趋势, 将很快发展起来。

更进一步, 可以根据斑块功能的相对重要值区 别出斑块在网络中是“源”还是“汇”, 如上述的 $C^{\mathrm{r}}$ 指 数, 当 $C^{r}>1$ 时, 斑块为 “源”, 当 $C^{r}<1$ 时, 斑块为 “汇” (Runge et al, 2006)。Pulliam (1988)最早从出生 率、移入率、死亡率和迁出率的角度定义了 “源” 或 “汇”，之后学者们多探索斑块尺度上“源”和“汇”的 动态属性(Pulliam, 1988; Donovan et al, 1995), 2000
年后有能力跟踪和模拟鸟类迁徙, “源” “汇”关系能 够在景观水平上被予以考虑, 就具有了更强烈的景 观生态学意义(Runge et al, 2006; Taylor \& Norris, 2010)。

鸟类迁徙网络中的“源” “汇”研究对“源” “汇” 景观生态理论有特殊的贡献。多种景观过程研究都 对“源” “汇”理论做出了贡献, 例如, 中国学者在研 究非点源的污染物运移过程中, 考虑了各类型斑块 在污染形成中的功能差异，从“源” “汇”景观理论角 度, 提出 “景观空间负荷比指数”, 突破了景观生态 学仅描述空间几何特征的瓶颈(陈利顶等, 2006)。这 种污染物的运移被动地决定于地形和景观类型等 特征、基本上是单向流动的; 动物移动过程则不同, 迁徙物种每年在越冬地和繁殖地之间往返迁徙, 种 群动态复杂多变，“源”和“汇”的关系非常复杂且具 有时空动态性。例如, 传统定义通常认为繁殖地是 “源” (有新个体出生), 非繁殖地是“汇”，但鸟类迁 徙时会在多个地点停歇, 形成复杂的网络, 非繁殖 地在迁徙种群动态中也起着必要、重要的作用, 不 能简单依据是否有新个体出生判断“源”或“汇” (Erickson et al, 2017)。动物种群的复杂动态性为 “源” “汇”景观理论提供了更大的研究空间，丰富了 这一理论。

\section{3 动物移动网络的连接度分析}

从动物移动的角度研究景观连接度, 落实了景 观功能连接度的概念 (Baguette \& Van Dyck, 2007)。Taylor等(1993)首次将景观连接度定义为动 物在离散的适宜生境斑块之间移动能力的度量, 之 后出现的多种定义都以此为基础(Kool et al, 2013)。 这个起始观点就特别强调利用动物种群特征和空 间行为规律才能研究景观连接度 (Taylor et al, 1993)。这种观点及以后以此观点为原则的研究案例 使得景观生态学最初提出的网络的概念和度量有 了真正的落脚点(Diniz et al, 2020), 并通过跟踪动 物轨迹获得了更多的属性特征, 推动了功能连接度 研究的发展。

对陆生动物而言, 地表景观特征是影响其运动 的主要因素之一, 通常利用地表景观特征模拟景观 连接度。最常用的模型是最小费用路径模型和电路 理论模型, 前者模拟动物通过不同景观类型的累计 成本加权距离(Etherington \& Holland, 2013), 后者 将阻力值对应为电阻值, 模拟电阻距离(Mcrae et al, 
2008; 宋利利和秦明周, 2016)。这两种模型都需要 同一个参数, 即不同景观类型的阻力值, 但是目前 该阻力值的设定普遍带有主观性, 极少依据实际的 动物移动特征(陈春娣等, 2017)。解决这个问题的办 法、也是未来的发展趋势就是要考虑动物实际运动 轨迹。实际的跟踪定位数据一方面有助于更加客观 地设定景观阻力值, 增强上述两种模型模拟的合理 性, 另一方面可以在考虑景观特征的基础上, 直接 计算景观连接度, 例如利用移步选择方程模型就能 够实现这种计算, 并能探讨连接度在季节及昼夜之 间的变化(Thurfjell et al, 2014; Martin et al, 2018)。

对长距离迁徙的鸟类而言, 不需要考虑两个生 境斑块之间的地表景观阻力, 只需要关注斑块上种 群特征的迁徙连接度 (degree of migratory connectivity) (Ambrosini et al, 2009; Poor et al, 2012)。迁徙连接度描述种群在不同季节之间空间利 用的一致性程度, 通常用两个迁徙季栖息地斑块上 种群之间的Mantel系数 $\left(\mathrm{r}_{\mathrm{m}}\right.$ )来表征(Marra et al, 2006; Cohen et al, 2018), 是典型的功能连接度。近5年, 由 于环志数据和跟踪定位数据的迅速积累和深度发 掘, 迁徙连接度研究迅速成为热点, 出现了为数不 少的针对水禽、鸣禽甚至猛禽等的研究案例 (Cormier et al, 2013; Hallworth et al, 2015; Finch et al, 2017; Sarà et al, 2019)。作为新兴的热点, 迁徙连接 度研究也面临着如何获得鸟类个体位置和种群数 量的困难(Hallworth et al, 2015), 目前最好的数据来 源是环志数据, 欧美国家在这方面有很大优势, 但 也不能完全满足需求, 正在寻求解决的途径, 例如 为了摆脱数据采样数量的限制, Cohen等(2018) 将 $\mathrm{r}_{\mathrm{m}}$ 指数扩展到利用种群转移概率进行估算。

\section{3 我国动物移动网络研究的现状、问题及 生态学发展的贡献}

我国学者克服研究基础薄弱、野外工作难度大 等种种困难, 针对动物移动网络开展研究, 取得了 巨大进步。但与国际水平相比尚显落后: (1)在动物 移动网络的构建模拟方面, 对于陆生动物, 多数研 究笼统地针对多种动物, 构建保护区或重要栖息地 之间的廊道(郭贤明等, 2015; 张玥等, 2020), 即使 针对某特定目标种的研究(龚明昊等, 2015), 也多仅 考虑地面景观特征, 利用最小阻力模型或者电路理 论模拟潜在廊道, 缺乏动物空间行为数据的支持
(Li et al, 2010; 侯宁等, 2014; 李维平等, 2017; 陈 强强等, 2019); 对于迁徙鸟类, 近几年开始利用 GPS跟踪数据构建迁徙网络(Wang et al, 2018; Deng et al, 2019), 但同时考虑栖息地景观特征和飞行特 征的种群水平迁徙路线的模拟暂时没有报道。(2) 在网络重要值评价方面, 利用中心性值评价结构重 要性以及利用种群特征评价节点的功能重要性都 非常少(Xu et al, 2020)。(3)在景观连接度研究方面, 针对陆生动物, 多利用地表阻力值或电路理论来度 量(吴昌广等, 2009; 薛亚东等, 2011; 刘世梁等, 2017; 李慧等, 2018), 尚未考虑动物实际运动轨迹; 针对鸟类, 迁徙网络的结构连接度研究很有限 $(\mathrm{Xu}$ et al, 2019), 功能连接度即迁徙连接度少有报道。

可以看出, 我国这三方面研究的落后之处也都 指向一个“焦点”: 未能考虑动物的空间行为特征, 并且这一问题还未被充分认识(单楠等, 2019)。该可 题产生的主要原因是目前我国动物空间行为研究 基础尚薄弱, 不能提供家域范围和行动距离等信息, 而这些重要空间行为信息的最主要来源是跟踪定 位数据, 因此以跟踪数据为基础的动物空间行为生 态研究就成为不能替代和逾越的内容。我国以无线 电跟踪(宋延龄等, 2000; 王文等, 2007; 刘雪华等, 2008)和GPS跟踪(杨红军等, 2006; 官天培等, 2015; 袁否等, 2015; Yuan et al, 2019)为数据源的兽类网络 研究比较有限; 鸟类GPS跟踪研究近几年迅速增加 (Wang et al, 2018; Deng et al, 2019; Xu et al, 2019, 2020), 尽管暂时尚不足以满足动物移动网络研究 发展的需求, 但也使得鸟类迁徙网络研究最有可能 成为我国近期动物移动网络研究的突破点。可以说, 源于跟踪数据的研究滞后已显现出对移动网络研 究的阻碍, 甚至成为发展的瓶颈。

国际上, 以跟踪数据为基础的动物空间行为生 态学研究非常扎实。例如, 陆生动物中仅鹿科动物 就有很多研究利用GPS跟踪数据估算其家域, 可提 供移动规律参数、用来构建和模拟移动网络及计算 迁徙连接度; 鸟类也有大量跟踪定位工作, 能够支 持重要值及迁徙连接度研究, 极大地促进了国际上 动物移动网络及相关领域研究的发展。在此基础上, Turner于2015年展望了未来25年的景观生态学研究 前景, 将 “多物种的相互作用和种内的变量”作为未 来六大热点研究方向之一, 意味着未来生物过程研 究将更加迅猛地发展(Turner, 2015)。我国若不能及 
时突破这一瓶颈，与国际水平之间的差距可能会继 续加大。

景观生态学自20世纪80年代末被引入我国, 至 今针对多种景观过程的研究都取得了丰硕的成果。 学者们结合各种具体过程研究了网络模拟、网络评 价以及景观连接度, 构建了景观网络研究的基本框 架，例如上面提到的污染物运移的“源” “汇”理论， 极大地丰富了我国景观生态学研究。但不得不说, 动物移动网络研究基本停留在空间结构关系阶段, 暂时尚未体现出这种独特的生物过程研究对我国 景观生态学的特殊贡献。

\section{4 结语}

景观生态学在其发展之初对网络的概念、网络 结构指数和景观连接度都做出了基本构想, 至今 30 多年间, 这些构想随着各种景观过程研究的深入被 逐渐落实并发展。其中, 动物移动形成的网络, 因 其节点或廊道上的属性独特丰富, 这些属性特征与 环境之间相互反馈、关系复杂, 给予了景观网络研 究极大的发展空间, 最大限度地实现了景观生态学 当初的构想, 并向着充分考虑动物空间行为特征的 方向发展, 在助推景观生态学发展中发挥着独特的 作用。可以说, 动物移动网络研究较全面地落实了 景观生态学中的景观网络理论, 是景观生态学深入 到过程研究的重要标志, 是“格局和过程”理论的经 典案例, 是持久不衰的研究议题, 一直以来在景观 生态学研究中熠熠生辉, 值得、也吸引着学者们更 加努力地探索。

我国的移动网络研究目前比较落后, 暂时未能 在助推我国景观生态学发展中起到应有的作用, 但 研究方向可以明确，即发展源于跟踪定位数据的动 物空间行为特征研究, 这将极大地推动动物移动网 络研究的发展, 最终为我国景观生态学发展做出其 特有的贡献。

\section{ORCID}

李月辉 (D) https://orcid.org/0000-0002-6476-868X 胡远满 (D) https://orcid.org/0000-0002-8017-5825

\section{参考文献}

Adriaensen F, Chardon JP, De Blust G, Swinnen E, Villalba S, Gulinck H, Matthysen E (2003) The application of 'least-cost' modelling as a functional landscape model. Landscape and Urban Planning, 64, 233-247.

Ambrosini R, Moller AP, Saino N (2009) A quantitative measure of migratory connectivity. Journal of Theoretical Biology, 257, 203-211.

Baguette M, Van Dyck H (2007) Landscape connectivity and animal behavior: Functional grain as a key determinant for dispersal. Landscape Ecology, 22, 1117-1129.

Baudry J, Merriam G (1988) Connectivity and connectedness: Functional versus structural patterns in landscapes. In: Connectivity in Landscape Ecology (ed. Schreiber KF), pp. 23-28. Münstersche Geogr, Arbeiten.

Bieri JA, Sample C, Thogmartin WE, Diffendorfer JE, Earl JE, Erickson RA, Federico P, Flockhart DTT, Nicol S, Semmens D, Skraber T, Wiederholt R, Mattsson BJ (2018) A guide to calculating habitat-quality metrics to inform conservation of highly mobile species. Natural Resource Modeling, 31, 1-46.

Blazquez-Cabrera S, Ciudad C, Gaston A, Simon MA, Saura S (2019) Identification of strategic corridors for restoring landscape connectivity: Application to the Iberian lynx. Animal Conservation, 22, 210-219.

Buhnerkempe MG, Webb CT, Merton AA, Buhnerkempe JE, Givens GH, Miller RS, Hoeting JA (2016) Identification of migratory bird flyways in North America using community detection on biological networks. Ecological Applications, 26, 740-751.

Chen CD, Jia ZY, Wu SJ, Tong XX, Zhou WZ, Chen RY, Zhang CL (2017) A bibliometric review of Chinese studies on the application of landscape connectivity. Acta Ecologica Sinica, 37, 3243-3255. (in Chinese with English abstract) [陈春娣，贾振毅，吴胜军，童笑笑，周文佐，陈若猗，张 超林 (2017) 基于文献计量法的中国景观连接度应用研 究进展. 生态学报, 37, 3243-3255.]

Chen LD, Fu BJ, Zhao WW (2006) Source-sink landscape theory and its ecological significance. Acta Ecologica Sinica, 26, 1444-1449. (in Chinese with English abstract) [陈利顶，傅伯杰，赵文武 (2006) “源” “汇”景观理论及其 生态学意义. 生态学报, 26, 1444-1449.]

Chen QQ, Li ML, Wang X, Wang P, Yang JW, Wang MY, Yang WK (2019) Identification of potential ecological corridors for Marco Polo sheep in Taxkorgan Wildlife Nature Reserve, Xinjiang, China. Biodiversity Science, 27, 186-199. (in Chinese with English abstract) [陈强强, 李美 玲, 王旭, 王鹏, 杨建伟, 汪沐阳, 杨维康 (2019) 新疆 塔什库尔干野生动物自然保护区马可波罗盘羊潜在生态 廊道识别. 生物多样性, 27, 186-199.]

Cohen EB, Hostetler JA, Hallworth MT, Rushing CS, Sillett TS, Marra PP (2018) Quantifying the strength of migratory connectivity. Methods in Ecology and Evolution, 9, 513-524.

Cooper NW, Ewert DN, Wunderle JM, Helmer EH, Marra PP (2019) Revising the wintering distribution and habitat use of 
the Kirtland's warbler using playback surveys, citizen scientists, and geolocators. Endangered Species Research, 38, 79-89.

Cormier RL, Humple DL, Gardali T, Seavy NE (2013) Light-level geolocators reveal strong migratory connectivity and within-winter movements for a coastal California Swainson's Thrush (Catharus ustulatus) population. The Auk, 130, 283-290.

Deng XQ, Zhao Q, Fang L, Xu ZG, Wang X, He HR, Cao L, Fox AD (2019) Spring migration duration exceeds that of autumn migration in Far East Asian Greater White-fronted Geese (Anser albifrons). Avian Research, 10, 319-329.

Diniz MF, Cushman SA, Machado RB, De Marco P (2020) Landscape connectivity modeling from the perspective of animal dispersal. Landscape Ecology, 35, 41-58.

Donovan TM, Thompson FR, Faaborg J, Probst JR (1995) Reproductive success of migratory birds in habitat sources and sinks. Conservation Biology, 9, 1380-1395.

Driezen K, Adriaensen F, Rondinini C, Doncaster CP, Matthysen E (2007) Evaluating least-cost model predictions with empirical dispersal data: A case-study using radiotracking data of hedgehogs (Erinaceus europaeus). Ecological Modelling, 209, 314-322.

Dutta T, Sharma S, Mcrae BH, Roy PS, Defries R (2016) Connecting the dots: Mapping habitat connectivity for tigers in central India. Regional Environmental Change, 16, 53-67.

Erickson RA, Diffendorfer JE, Norris DR, Bieri JA, Earl JE, Federico P, Fryxell JM, Long KR, Mattsson BJ, Sample C (2017) Defining and classifying migratory habitats as sources and sinks: The migratory pathway approach. Journal of Applied Ecology, 55, 108-117.

Estrada E, Bodin O (2008) Using network centrality measures to manage landscape connectivity. Ecological Applications, 18, 1810-1825.

Etherington TR, Holland EP (2013) Least-cost path length versus accumulated-cost as connectivity measures. Landscape Ecology, 28, 1223-1229.

Finch T, Butler SJ, Franco AMA, Cresswell W (2017) Low migratory connectivity is common in long-distance migrant birds. Journal of Animal Ecology, 86, 662-673.

Forman RTT (1995) Some general principles of landscape and regional ecology. Landscape Ecology, 10, 133-142.

Forman RTT, Baudry J (1984) Hedgerows and hedgerow networks in landscape ecology. Environmental Management, 8, 495-510.

Forman RTT, Godron M (1986) Landscape Ecology. John Wiley \& Sons, New York.

Gong MH, Ouyang ZY, Xu WH, Song YL, Dai B (2015) The location of wildlife corridors under the impact of road disturbance: Case study of a giant panda conservation corridor. Acta Ecologica Sinica, 35, 3447-3453. (in Chinese with English abstract) [龚明昊, 欧阳志云, 徐卫华, 宋延
龄, 戴波 (2015) 道路影响下野生动物廊道的选址一 以大熊猫保护廊道为例. 生态学报, 35, 3447-3453.]

Guan TP, Ge BM, Chen LM, You ZQ, Tang ZH, Liu H, Song YL (2015) Home range and fidelity of Sichuan takin. Acta Ecologica Sinica, 35, 1862-1868. (in Chinese with English abstract) [官天培, 葛宝明, 谌利民, 游章强, 唐中海, 刘 吴, 宋延龄 (2015) 四川羚牛的家域与忠诚度. 生态学 报, 35, 1862-1868.]

Guillemain M, Calenge C, Champagnon J, Hearn R (2017) Determining the boundaries of migratory bird flyways: A Bayesian model for Eurasian teal Anas crecca in western Europe. Journal of Avian Biology, 48, 1331-1341.

Guo XM, Wang LX, Yang ZB, Zhao JW, Yang HP (2015) Large design case study of wildlife migration corridor-A case study for development of biodiversity conservation corridor between Mengla Sub-reserve and Mengyang Sub-reserve. Journal of Shandong Forestry Science and Technology, 45(1), 1-7. (in Chinese with English abstract) [郭贤明，王兰新，杨正斌，赵建伟，杨鸿培 (2015) 大型 野生动物迁徙廊道设计案例分析一以预腊-预养保护区 间廊道设计为例. 山东林业科技, 45(1), 1-7.]

Hallworth MT, Sillett TS, Van Wilgenburg SL, Hobson KA, Marra PP (2015) Migratory connectivity of a Neotropical migratory songbird revealed by archival light-level geolocators. Ecological Applications, 25, 336-347.

Hanski I (1998) Metapopulation dynamics. Nature, 396, 41-49.

Hou N, Dai Q, Ran JH, Jiao YY, Cheng Y, Zhao C (2014) A corridor design for the giant panda in the Niba Mountain of China. Chinese Journal of Applied and Environmental Biology, 20, 1039-1045. (in Chinese with English abstract) [侯宁, 戴强, 由江洪, 焦迎迎, 程勇, 赵成 (2014) 大相 岭山系泥巴山大熊猫生境廊道设计. 应用与环境生物学 报, 20, 1039-1045.]

Iwamura T, Fuller RA, Possingham HP (2014) Optimal management of a multispecies shorebird flyway under sea-level rise. Conservation Biology, 28, 1710-1720.

Keeley ATH, Beier P, Keeley BW, Fagan ME (2017) Habitat suitability is a poor proxy for landscape connectivity during dispersal and mating movements. Landscape Urban Planning, 161, 90-102.

Kerr B, Neuhauser C, Bohannan BJM, Dean AM (2006) Local migration promotes competitive restraint in a host-pathogen 'Tragedy of the Commons'. Nature, 442, 75-78.

Kneitel JM, Miller TE (2003) Dispersal rates affect species composition in metacommunities of Sarracenia purpurea inquilines. The American Naturalist, 162, 165-171.

Knight SM, Bradley DW, Clark RG, Gow EA, Belisle M, Berzins LL, Blake T, Bridge ES, Burke L, Dawson RD, Dunn PO, Garant D, Holroyd GL, Hussell DJT, Lansdorp O, Laughlin AJ, Leonard ML, Pelletier F, Shutler D, Siefferman L, Taylor CM, Trefry HE, Vleck CM, Vleck D, Winkler DW, Whittingham LA, Norris DR (2018) Constructing and evaluating a continent-wide migratory 
songbird network across the annual cycle. Ecological Monographs, 88, 445-460.

Kool JT, Moilanen A, Treml EA (2013) Population connectivity: Recent advances and new perspectives. Landscape Ecology, 28, 165-185.

Lapoint SD, Gallery P, Wikelski M, Kays R (2013) Animal behavior, cost-based corridor models, and real corridors. Landscape Ecology, 28, 1615-1630.

LaRue MA, Nielsen CK (2008) Modelling potential dispersal corridors for cougars in midwestern North America using least-cost path methods. Ecological Modelling, 212, 372-381.

Li H, Li L, Wu GS, Zhou Y, Li WW, Mei ZW (2018) Analysis of the landscape-level connectivity of the Yunnan snub-nosed monkey habitat based on circuit theory. Acta Ecologica Sinica, 38, 2221-2228. (in Chinese with English abstract) [李慧, 李丽, 吴巩胜, 周跃, 李雯雯, 梅泽文 (2018) 基于电路理论的滇金丝猴生境景观连通性分析. 生态学报, 38, 2221-2228.]

Li HL, Li DH, Li T, Qiao Q, Yang J, Zhang HM (2010) Application of least-cost path model to identify a giant panda dispersal corridor network after the Wenchuan earthquake-Case study of Wolong Nature Reserve in China. Ecological Modelling, 221, 944-952.

Li WP, Bao H, Zhang MH (2017) Habitat analysis and design of potential ecological corridors for Amur tiger in Northeastern China. Acta Theriologica Sinica, 37, 317-326. (in Chinese with English abstract) [李维平, 包衡, 张明海 (2017) 中国东北虎栖息地分析与潜在生态廊道构建. 兽 类学报, 37, 317-326.]

Li XZ, Xiao DN, Hu YM, Wang XL (2001) Effect of wetland landscape pattern on nutrient reduction in the Liaohe Delta. Acta Geographica Sinica, 56, 32-43. (in Chinese with English abstract) [李秀珍, 肖笃宁, 胡远满, 王宪礼 (2001) 辽河三角洲湿地景观格局对养分去除功能影响的 模拟. 地理学报, 56, 32-43.]

Liu SL, Hou XY, Yin YJ, Cheng FY, Zhang YQ, Dong SK (2017) Research progress on landscape ecological networks. Acta Ecologica Sinica, 37, 3947-3956. (in Chinese with English abstract) [刘世梁, 侯笑云, 尹艺洁, 成方妍, 张月 秋, 董世鬼 (2017) 景观生态网络研究进展. 生态学报, 37, 3947-3956.]

Liu XH, Wang T, Wang PY, Yang J (2008) Application research of radio-tracking data on giant panda movements in Wolong Nature Reserve, China. Acta Theriologica Sinica, 28, 180-186. (in Chinese with English abstract) [刘雪华, 王亭, 王鹏彦, 杨健 (2008) 无线电颈圈定位数据应用于 卧龙大熊猫移动规律的研究. 兽类学报, 28, 180-186.]

Marra PP, Norris DR, Haig SM, Webster MS, Royle JA (2006) Migratory connectivity. In: Conservation Connectivity (eds Crooks K, Sanjayan MA), pp. 157-183. Cambridge University Press, New York.

Martin J, Vourch G, Bonnot N, Cargnelutti B, Chaval Y,
Lourtet B, Goulard M, Hoch T, Plantard O, Hewison AJM (2018) Temporal shifts in landscape connectivity for an ecosystem engineer, the roe deer, across a multiple-use landscape. Landscape Ecology, 33, 937-954.

Mcrae BH, Dickson BG, Keitt TH, Shah VB (2008) Using circuit theory to model connectivity in ecology, evolution, and conservation. Ecology, 89, 2712-2724.

Merriam G (1984) Connectivity: A fundamental ecological characteristic of landscape pattern. In: Proceedings of the First International Seminar on Methodology in Landscape Ecological Research and Planning (eds Brandt J, Agger P), pp. 5-15. Roskilde Universitetsforlag GeoRuc, Roskilde.

Nicol S, Wiederholt R, Diffendorfer JE, Mattsson BJ, Thogmartin WE, Semmens DJ, Lopez-Hoffman L, Norris DR (2016) A management-oriented framework for selecting metrics used to assess habitat- and path-specific quality in spatially structured populations. Ecological Indicators, 69, 792-802.

Palm EC, Newman SH, Prosser DJ, Xiao XM, Ze L, Batbayar N, Balachandran S, Takekawa JY (2015) Mapping migratory flyways in Asia using dynamic Brownian bridge movement models. Movement Ecology, 3, art3.

Panzacchi M, Van Moorter B, Strand O, Saerens M, Kivimaki I, Clair CCS, Herfindal I, Boitani L (2016) Predicting the continuum between corridors and barriers to animal movements using Step Selection Functions and Randomized Shortest Paths. Journal of Animal Ecology, 85, 32-42.

Poor EE, Loucks C, Jakes A, Urban DL (2012) Comparing habitat suitability and connectivity modeling methods for conserving pronghorn migrations. PLoS ONE, 7, e49390.

Pulliam HR (1988) Sources, sinks, and population regulation. The American Naturalist, 132, 652-661.

Pullinger MG, Johnson CJ (2010) Maintaining or restoring connectivity of modified landscapes: Evaluating the least-cost path model with multiple sources of ecological information. Landscape Ecology, 25, 1547-1560.

Risser PG, Karr JR, Forman RTT (1984) Landscape Ecology: Directions and Approaches. Illinois Natural History Survey Special Publication, Urbana.

Runge JP, Runge MC, Nichols JD (2006) The role of local populations within a landscape context: Defining and classifying sources and sinks. The American Naturalist, 167, 925-938.

Sample C, Bieri JA, Allen B, Dementieva Y, Carson A, Higgins C, Piatt S, Qiu S, Stafford S, Mattsson BJ, Semmens DJ, Thogmartin WE, Diffendorfer JE (2019) Quantifying source and sink habitats and pathways in spatially structured populations: A generalized modelling approach. Ecological Modelling, 407, 108715.

Sarà $\mathrm{M}$, Bondì $\mathrm{S}$, Bermejo $\mathrm{A}$, Bourgeois $\mathrm{M}$, Bouzin $\mathrm{M}$, Bustamante J, Puente J, Evangelidis A, Frassanito A, Fulco E, Giglio G, Gradev G, Griggio M, López-Ricaurte L, Kordopatis P, Marin S, Martínez J, Mascara R, Mellone U, 
Pellegrino SC, Pilard P, Podofillini S, Romero M, Gustin M, Saulnier N, Serra L, Sfougaris A, Urios V, Visceglia M, Vlachopoulos K, Zanca L, Cecere JG, Rubolini D (2019) Broad-front migration leads to strong migratory connectivity in the lesser kestrel (Falco naumanni). Journal of Biogeography, 46, 13713.

Shan N, Zhou KX, Pan Y, Tang FK (2019) Research advances in design methods of biodiversity conservation corridors. Acta Ecologica Sinica, 39, 411-420. (in Chinese with English abstract) [单楠, 周可新, 潘扬, 唐夫凯 (2019) 生 物多样性保护廊道构建方法研究进展. 生态学报, 39, 411-420.]

Sheehy J, Taylor CM, Norris DR (2011) The importance of stopover habitat for developing effective conservation strategies for migratory animals. Journal of Ornithology, 152, 161-168.

Song LL, Qin MZ (2016) Identification of ecological corridors and its importance by integrating circuit theory. Chinese Journal of Applied Ecology, 27, 3344-3352. (in Chinese with English abstract) [宋利利, 秦明周 (2016) 整合电路 理论的生态廊道及其重要性识别. 应用生态学报, 27, 3344-3352.]

Song YL, Zeng ZG, Zhang J, Wang XJ, Gong HS, Wang KW (2000) Home range of golden takin (Budorcas taxicolor bedfordi) in Foping Nature Reserve, Shaanxi, China. Acta Theriologica Sinica, 20, 241-249. (in Chinese with English abstract) [宋延龄, 曾治高, 张坚, 王学杰, 巩会生, 王宽 武 (2000) 秦岭羚牛的家域研究. 兽类学报, 20 , 241-249.]

Taylor CM, Hall RJ (2012) Metapopulation models for seasonally migratory animals. Biology Letters, 8, 477-480.

Taylor CM, Norris DR (2010) Population dynamics in migratory networks. Theoretical Ecology, 3, 65-73.

Taylor PD, Fahrig L, Henein K, Merriam G (1993) Connectivity is a vital element of landscape structure. Oikos, 68, 571-573.

Thogmartin WE (2010) Modeling and mapping Golden-winged Warbler abundance to improve regional conservation strategies. Avian Conservation and Ecology, 5, art12.

Thurfjell H, Ciuti S, Boyce MS (2014) Applications of step-selection functions in ecology and conservation. Movement Ecology, 2, 1-12.

Turner MG (1989) Landscape ecology: The effect of pattern on process. Annual Review of Ecology and Systematics, 20, 171-197.

Turner MG (2015) Twenty-five Years of United States Landscape Ecology: Looking Back and Forging Ahead. Springer, New York.

Van Toor ML, Kranstauber B, Newman SH, Prosser DJ, Takekawa JY, Technitis G, Weibel R, Wikelski M, Safi K (2018) Integrating animal movement with habitat suitability for estimating dynamic migratory connectivity. Landscape Ecology, 33, 879-893.
Vanthomme HPA, Nzamba BS, Alonso A, Todd AF (2019) Empirical selection between least-cost and current-flow designs for establishing wildlife corridors in Gabon. Conservation Biology, 33, 329-338.

Wang W, Zhang J, Ma JZ, Liu HB (2007) Analysis on wild boars home ranges in south Lesser Khingan Mountains. Acta Theriologica Sinica, 27, 257-262. (in Chinese with English abstract) [王文, 张静, 马建章, 刘海波 (2007) 小 兴安岭南坡野猪家域分析. 兽类学报, 27, 257-262.]

Wang X, Cao L, Batbayar N, Fox AD (2018) Variability among autumn migration patterns of Mongolian Common Shelducks (Tadorna tadorna). Avian Research, 9, 71-81.

Watts K, Eycott AE, Handley P, Ray D, Humphrey JW, Quine CP (2010) Targeting and evaluating biodiversity conservation action within fragmented landscapes: An approach based on generic focal species and least-cost networks. Landscape Ecology, 25, 1305-1318.

Webster MS, Marra PP, Haig SM, Bensch S, Holmes RT (2002) Links between worlds: Unraveling migratory connectivity. Trends in Ecology \& Evolution, 17, 76-83.

Wiederholt R, Mattsson BJ, Thogmartin WE, Runge MC, Diffendorfer JE, Erickson RA, Federico P, Lopez-Hoffman L, Fryxell JM, Norris DR, Sample C (2018) Estimating the per-capita contribution of habitats and pathways in a migratory network: A modelling approach. Ecography, 41, 815-824.

Wu CG, Zhou ZX, Wang PC, Xiao WF, Teng MJ, Peng L (2009) Evaluation of landscape connectivity based on least-cost model. Chinese Journal of Applied Ecology, 20, 2042-2048. (in Chinese with English abstract) [吴昌广, 周 志翔, 王鹏程, 肖文发, 滕明君, 彭丽 (2009) 基于最小 费用模型的景观连接度评价. 应用生态学报, 20, 2042-2048.]

Wu JG (2000) Landscape ecology-Concepts and theories. Chinese Journal of Ecology, 19, 42-52. (in Chinese with English abstract) [邬建国 (2000) 景观生态学——概念与 理论. 生态学杂志, 19, 42-52.]

Xu Y, Si Y, Takekawa J, Liu Q, Prins HHT, Yin S, Prosser DJ, Gong P, de Boer WF (2020) A network approach to prioritize conservation efforts for migratory birds. Conservation Biology, 34, 416-426.

Xu Y, Si Y, Wang Y, Zhang Y, Prins HHT, Cao L, de Boer WF (2019) Loss of functional connectivity in migration networks induces population decline in migratory birds. Ecological Applications, 29, e01960.

Xue YD, Li L, Li DQ, Wu GS, Zhou Y, Lü XX (2011) Analysis of habitat connectivity of the Yunnan snub-nosed monkeys (Rhinopithecus bieti) using landscape genetics. Acta Ecologica Sinica, 31, 5886-5893. (in Chinese with English abstract) [薛亚东, 李丽, 李迪强, 吴巩胜, 周跃, 吕告喜 (2011) 基于景观遗传学的滇金丝猴栖息地连接 度分析. 生态学报, 31, 5886-5893.]

Yang HJ, Zhang HH, Dou HS, Shen XQ, Yan WB, Zhang Y, 
Gui MQ, Bao MR, Chen JRMT (2006) Home range and habitat utilization of wolves during winter in Hulunbeir, Inner Mongolia. Journal of Northeast Forestry University, 34(4), 52-53. (in Chinese with English abstract) [杨红军, 张洪海, 赎华山, 沈秀清, 颜文博, 张友, 桂满全, 包牧 仁, 陈吉日木图 (2006) 呼伦贝尔草原冬季狼的巢域和 生境利用. 东北林业大学学报, 34(4), 52-53.]

Yuan B, Xie S, Liu B, Xue D, Sun D (2019) Differential movement pattern of Père David's deer associated with the temporal rhythm using GPS collar fix. Global Ecology and Conservation, 18, e00641.

Yuan L, Ma H, Cheng Y, Yang H, Sagen GL, Yadamsuren A, Zhu HY, Ma YQ, Wang JL, Zhang S, Ma XY, Diao QA, Liu SC (2015) Qualitative study of wild camels (Camelus ferus) home range in Lop Nur, China. Biodiversity Science, 23, 314-320. (in Chinese with English abstract) [袁否, 马浩, 程芸, 杨欢, 萨根古丽, Yadamsuren A, 朱海涌, 马友青, 王建林, 张炼, 马杏叶, 刀庆安, 刘少创 (2015) 罗布泊 野骆驼的家域特征及其意义. 生物多样性, 23, 314-320.]

Zeller KA, Mcgarigal K, Beier P, Cushman SA, Vickers TW, Boyce WM (2014) Sensitivity of landscape resistance estimates based on point selection functions to scale and behavioral state: Pumas as a case study. Landscape Ecology, 29, 541-557.

Zeller KA, Mcgarigal K, Cushman SA, Beier P, Vickers TW, Boyce WM (2016) Using step and path selection functions for estimating resistance to movement: Pumas as a case study. Landscape Ecology, 31, 1319-1335.

Zhang N (2014) Landscape Ecology. Science Press, Beijing. (in Chinese) [张娜 (2014) 景观生态学. 科学出版社, 北京.]

Zhang Y, Xu DY, Li X, Zhang XY, Wang XB, Wang SQ (2020) Construction of ecological corridors and identification of key nodes in the core area of China-Laos transportation corridors. Acta Ecologica Sinica, 40, 1933-1943. (in Chinese with English abstract) [张玥, 许端 阳, 李霞, 张晓宇, 王小博, 王绍强 (2020) 中-老交通走 廊核心区生态廊道构建与关键节点识别. 生态学报, 40, 1933-1943.] 